\title{
A Composite Mobility Model for Ad Hoc Networks in Disaster Areas
}

\author{
Stéphane Pomportes ${ }^{1}$, Joanna Tomasik ${ }^{2}$, Véronique Vèque ${ }^{1}$ \\ ${ }^{1}$ Université de Paris-Sud, Institut d'Electronique Fondamentale, Orsay Cedex, France \\ ${ }^{2}$ SUPELEC Systems Sciences (E3S), Computer Science Department, Gif sur Yvette, France
}

Correspondence: Joanna Tomasik, joanna.tomasik@supelec.fr

Manuscript communication: received 17 December 2010, accepted 12 January 2011

\begin{abstract}
Ad hoc network is an essential means of communication in disaster scenes. Validation of new communication protocols for these networks requires exhaustive simulation runs because real life experiences can be performed exclusively during expensive civil protection exercises. The necessity of these simulations calls for realistic and reliable mobility models which take into account numerous aspects of rescue worker interventions. We propose a composite model of mobility for disaster scenarii which includes a realistic model of human displacement, team mobility, and obstacle avoidance. The comparison of its performance with those of other available models is encouraging. We believe that it can improve the veracity of rescue operation simulations.
\end{abstract}

Keywords- Ad hoc network, mobility model.

\section{INTRODUCTION}

Natural or man-made, disasters, like earthquake or flooding, may cause destruction of both the wired and wireless infrastructures in the affected area. Such damage precludes the utilization of accustomed means of communication and makes mobile ad hoc networks indispensable. Search and rescue teams consisting of, for example, medical staff, firemen, policemen, etc. operating on the devastated terrain communicate with one another and serve as routers assuring communications between colleagues. The consequence of this fact is that emitters, receivers, and routers are carried by humans and they follow human movements. Movements of rescue workers on a disaster scene are determined by various factors particular for a given event (localization of fire centers, injured persons, deployed medical aid points, etc.). They can also be strategic (organization of rescue workers in teams, hierarchy imposed by commandment, etc.), and geographical (physical obstacles, routes, etc.). Mobility models proposed for simulation of disaster areas in order to evaluate the performance of novel technical solutions have to take these elements into account. As real-life experiences concerning performance evaluation are too expensive (or even unrealizable), the principal means of evaluation is simulation. For this reason, well adapted and realistic mobility models have a crucial influence upon the quality of results estimated by simulations

In the face of the facts stated above we decided to propose a mobility model taking into account varied aspects of rescue worker movements. The work presented was done within the context of the RAF project which is also one of the projects of the "SYSTEM@TIC PARIS-REGION competitiveness cluster". In Section 2 we survey mobility models which can be found in the scientific literature. These models are discussed according to their characteristic features. In this discussion we point out their weak points. In Section 3 we present our composite model explaining our choice of the individual elements in detail. We explain namely the modifications of the Voronoï diagram which we propose in order to adapt it so that it could trace routes in urban areas with various obstacle configurations. Section 4 contains the comparisons of the performance of our model with others in the context of the two different scenarii. Finally, we conclude our work and outline the perspectives.

\section{Related Work}

\subsection{Movement Models}

The authors of [1] reviewed more than 150 scientific papers concerning MANET networks which had been published between 2000 and 2005 in order to compare their evaluation methods. The study results show that for most of them (75.5\%) simulations had been used to evaluate the research. The majority of papers $(73.8 \%)$ which took into account agent mobility use the well known Random Way-Point (RWP) [2]. The success of this model can be explained by its simplicity and its implementation in widely used simulators (ns-2). Yet, the model, besides its lack of realism, has many other undesirable properties highlighted in numerous studies: a high concentration of nodes in the simulation area [3-5], oscillations of the number of neighbors per node [6], and a decrease in the average speed of nodes over time. This last aspect is fully treated in [7] which shows that the speed decays quickly to reach the minimum 
allowed speed (when $V_{\min }=0$, the network becomes static).

Many works, based on an analysis of real traces, try to find statistical characteristics of human mobility. Some of these studies stress that the important elements of human mobility, such as the length of human displacement between two stops (called the flight length) [8-10], the pause time $[10,11]$, and the inter-contact time [12,13], can be approximated by a power law.

Some researchers based their works on the above results and proposed new models taking advantage of a Levy distribution which is a member of the power law family. The authors of [10] gathered many human displacement traces in varied contexts. They also proposed a new mobility model in which the flight length and the pause time follow some distribution of the power law family. In this model, the displacement of agents is defined by a sequence of steps. Each step is characterized by four variables: flight length $(l)$, pause time $\left(\Delta t_{p}\right)$, flight time $\left(\Delta t_{f}\right)$, and direction $(\theta)$.

Flight lengths and pause times follow a Levy distribution, which can be approximated by $\frac{1}{x^{\alpha+1}}$ and $\frac{1}{x^{\beta+1}}$, respectively, and with $0<\alpha, \beta<2$. The flight time is proportional to flight length following the formula: $\Delta t_{f}=k l^{1-\rho}, 0 \leq \rho \leq 1$, where $k$ and $\rho$ are constant parameters of the simulation. The parameter $k$ is a scale factor between flight length and flight time. If $\rho$ is 0 , flight time and flight length are proportional, thus velocity is constant. When $\rho$ is 1 , flight time is constant, thus velocity and flight. The direction $\vartheta$ is chosen uniformly from 0 to $360^{\circ}$.

According to their comparison of the traces generated by the model proposed in [10] and the real ones, a Levy distribution is the most adapted to represent human mobility. To the best of our knowledge, Levy based models have not yet been used in the context of civil defense scenarii. Another important property of rescuers movement is that they move in a group and and their way to move depends on the operation area.

\subsection{Models with Spatial Dependencies}

2.2.1 Group Mobility: As rescue workers operate mainly in teams, a mobility model should take this property into account. Movements of group members have a strong impact on communications (long lifetime of links, presence of clique [14], etc.). The Reference Point Group Mobility (RPGM) [15] model is focused on this aspect of mobility. According to its definition, mobile agents are divided into several groups. Each group has a leader who can be either a member of the group or a virtual point. In the studied context, the assumption that the leader is a member of its team is very plausible. The movement of the leader defines the displacement of an entire group. Each member of a group has, however, a certain freedom in the choice of his own motion relative to the leader's action. The authors of RPGM proposed RWP as a leader mobility model.

2.2.2 Obstacle Avoidance Models: In the mobility models discussed above, the simulation area is considered fully usable. In reality, there are obstacles such as ponds, buildings or debris of any size in a disaster area. These obstacles have a direct impact on the mobile agents who must adapt their trajectory to circumvent them. To overcome these obstacles, it is possible to use road maps containing all paths to get around the area of simulation while avoiding obstacles. The visibility graph [16] is another solution used in [7]. This approach provides the shortest path to the destination while avoiding obstacles. Nevertheless, this solution puts the mobile agents very close to the obstacles and, in some situations, such as the presence of burning objects or unstable buildings, it is not realistic to allow the rescue workers to walk close to dangerous obstacles. The authors of [17] proposed another solution, based on the Voronoï diagram, in which the obstacles are modeled as rectangles. The Voronoï diagram is built taking the obstacle corners as reference points. The boundaries between Voronoï cells are then used as a road map on which the agents can move to avoid obstacles. As we observed, when applying a Voronoï diagram for our modeling purposes, the map generated by Voronoï cell borders may be unrealistic for certain obstacle configurations, namely long obstacles parallel to one another with a short distance between them. In such cases modeled mobile agents have a tendency to envelop obstacles, keeping an unreasonably wide distance from these obstacles.

\subsection{Composite Models}

As introduced previously, movements of rescue workers are complex and the modeling process has to take into account a lot of features in a composite model. Recent studies suggest combining several models of mobility to create a more realistic macro-model.

2.3.1 Disaster Area (DA) Mobility Model: In [7], the authors studied the displacement of civil protection forces in real life exercises and developed a corresponding model. In this model, the simulation area is divided into sub-areas. Mobile agents are divided into several categories (civil protection forces including rescue teams and fire brigades) in order to reflect the specificity of each one (speed, area of intervention, etc.). A visibility graph is used to avoid obstacles. Nevertheless, some aspects are not yet realistic. The Group Mobility for example is not taken into account and the mobility of agents in each sub-area is determined by RWP. We decided not to use the visibility graph method because we are convinced that the rescue workers' security is more important than the length of their displacements in a disaster context. For this reason, agents opt for a longer path to avoid being too close to buildings.

2.3.2 Mission Critical Mobile (MCM) Model: The authors of [18] presented another combined model: Mission Critical Mobility. This model is similar to the previous one regarding management of obstacles and of agents' movements which follow a modified RWP. The RPGM model is used to represent the group mobility. The model discussed incorporates the propagation model defined in [17]. It does not include, however, the 


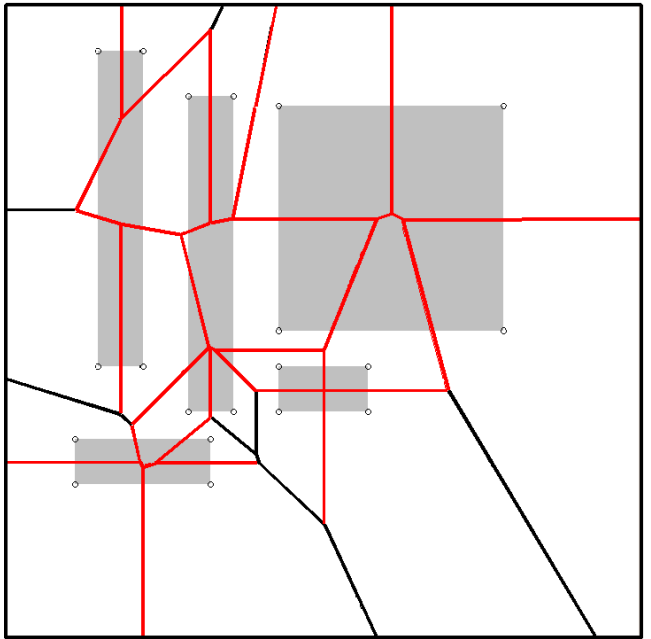

Figure 1. Original Voronoï diagram.



Figure 2. Modified Voronoï diagram. division of the simulation area into specific sub-areas and the choice of a zero minimum speed may cause the speed decaying problems mentioned in point II.A. In the next section, we introduce our composite model.

\section{Our Composite Mobility Model}

Most existing models, even realistic ones, use the RWP model despite the problems associated with its use. In this section we propose a composite model, based on a power-law model instead of the RWP one, which we believe particularly appropriate for simulations of disaster areas. Similarly to the models cited in the previous section, our composite model is based on existing ones. To obtain a sufficient level of realism, it integrates the essential features of a disaster scene: obstacle avoidance, group mobility, leader displacement more representative for human behavior than the problematic RWP, and mobility self-adaptive to the urban and rural areas of simulation.

In a nutshell, our model uses the following components. For the group mobility, this model uses the RPGM presented above. For a better realism, the RWP is replaced by the Levy-Walk model, presented in [10]. Concerning obstacle avoidance, our model integrates the solution based on a Voronoï diagram.

As mentioned above (Section II.B.2) routes which are derived from a Voronoï diagram, created with obstacle corners taken as reference points, do not always lead to a realistic trace map. To get round this weakness, we propose the following modification to the existing approach. Firstly, we introduce supplementary reference points. These additional points are projections of the obstacle corners on the edges of all the other obstacles. This first change creates paths which allow mobile agents "to worm" between obstacles. Secondly, we add the reference points on the border of the simulation area, that make a projection of the obstacle corners. This second change allows us to introduce paths permitting mobile agents to pass around the obstacles close to the area border. In Figures 1 and 2 we present the classical and the modified Voronoï diagrams for a given obstacle map.

The use of a Voronoï diagram, which provides a map indicating to the agents the paths avoiding the obstacles, may seem incompatible with the use of a Levy walk. In order to preserve the latter model, the Voronoï diagram is used only when an obstacle is present on the route proposed by Levy-walk. When an obstacle is on a path followed by an agent, the path is modified in order to reach the closest path on the corresponding Voronoï diagram and bypass the obstacle. To avoid disrupting the power-law distribution, the speed and flight length are conserved. When the agents bypass the obstacle or move a distance equals to the flight length, our model changes and becomes a "classical" model of movement, the RPGM and Levy-walk models. We precise that if a probabilistic draw would displace an agent out of the scope of the simulation area, it is repeated (length and rotation) until a new agent position is within the area ranges.

A mobile agent who is forced to avoid an obstacle looks for a shortest path between its current position and its destination among the different paths of the road map offered by the Voronoï diagram. The procedure of finding a shortest path starts by representing possible paths in an undirected weighted graph. Initially, the vertices of this graph are the crossing points of the Voronoï cells and its edges are the Voronoï cell borders. The edge weight is its length according to the Euclidean metric. Afterward, the starting and the destination points are added to the graph vertex set. The agent's starting and final points attached to the graph by the new edges which are introduced between each of them and all other vertex of the graph if and only if these edges do not cross any obstacle. The Dijkstra's algorithm [19] is used to find a shortest path between agent's initial and final position. The example of a possible path chosen by an agent starting from the point $A$ and arriving to the point $B$ in the given map is presented in Figure 3.

A great difficulty in introducing a group mobility model into a simulation scene with obstacles is that 




Figure 3. Path chosen by an agent starting from the point $\mathrm{A}$ and arriving to the point $\mathrm{B}$. The attachment points to the roadmap are a (for $\mathrm{A}$ ) and $\mathrm{b}$ (for B). This path circumvents the obstacles $\mathrm{X}, \mathrm{Y}$ and $\mathrm{Z}$.

group members accompanying their leader, in some rare cases, seem to be moving through obstacles. This undesired feature needs to be corrected locally by limiting the radius of an offset allowed around the leader according to the RPGM model. In a rural context the model only rarely uses a Voronoï diagram. In an urban setting characterized by many buildings a Voronoï diagram is used frequently. As the modified Voronoï diagram produces a fairly accurate map (diagram paths pass between each building as roads do in real life), the movement will be fairly realistic.

\section{Composite Model Evaluation}

In this section, we evaluate various aspects of our model. Firstly, we show that, in contrast to the RWP model, the Levy-walk does not tend to concentrate the nodes in the center of the simulation area. Secondly, we compare the distribution of the node degree, i.e., the number of neighbors, of our model to the distribution for the RPGM, RWP and Levy-walk models. The node degree is an important metric for performance of a number of routing protocols. Thirdly, we focus on the impact of obstacles on our mobility model. Thus we compute the average node degree, and both the contact and inter-contact times.

\subsection{Comparison of the Concentration of Nodes between the RWP Model and the Levy-walk Model}

Firstly we analyze the Levy walk model which is part of our composite model. One of the well known weaknesses of RWP (Section II.A) is due to a fact that mobile agents following this model have a tendency to group in the center of the simulation area. The value of Levy walk parameters are $\alpha=1.5, \beta=1$, and according to the results of [10], $k=18.72, \rho=0.79$ for small displacements (flightLength $<500 \mathrm{~m}$ ) and $k=1.37$, $\rho=0.36$ otherwise. These settings allow us not only to introduce more realistic human movements but also



Figure 4. Concentration of nodes which move according to RWP model in the simulation area.

Levy walk



Figure 5. Concentration of nodes which move according to Levy model in the simulation area.

to avoid this undesired concentration. To visualize the concentration, we introduce a measurement relative to the uniform distribution of agents on a simulation area without obstacles (400 agents, $800 \times 800$ simulation area). Let $\overline{N A}$ be the average number of agents who move according to a mobility model in a square unit. Let $\bar{U}$ be the mean number of agents whose position is chosen according to the uniform distribution in a square unit. We define the concentration metric as $\mathrm{C}=$ $\frac{\overline{N A}}{\bar{U}}$. Figures 4 and 5 present the measurement, using the concentration metric C, for both RWP and Levywalk. We precise that if a probabilistic draw should displace an agent out of the scope of the simulation area, it is repeated (both length and direction) until a new agent position is within the area ranges. The Levy walk model offers an "almost uniform" distribution in an area without obstacles. For a Levy walk model, the concavities along the simulation area borders are caused by a "pushing away" effect caused by these boundaries.

\subsection{Impact of our Model on Node Degree for Team Size Equal to 10}

In our following experiments we used a population of 1000 mobile agents. The impact of the mobility of agents (following RPGM, RWP, Levy or our model) on the average node degree in the area without obstacles (rural scenario) is presented in Figure 6. As we can notice, the impact of the group movement on the average degree in our model and in RPGM is exhibited for the 


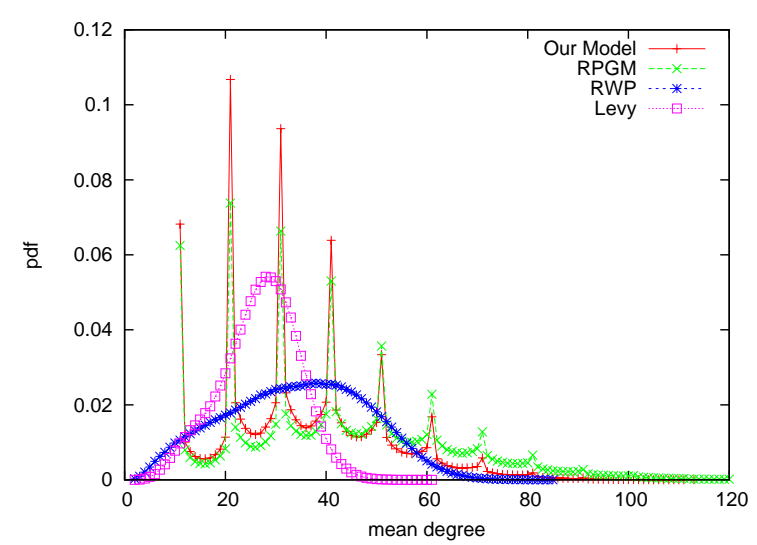

Figure 6. pdf of node degree for different mobility models.

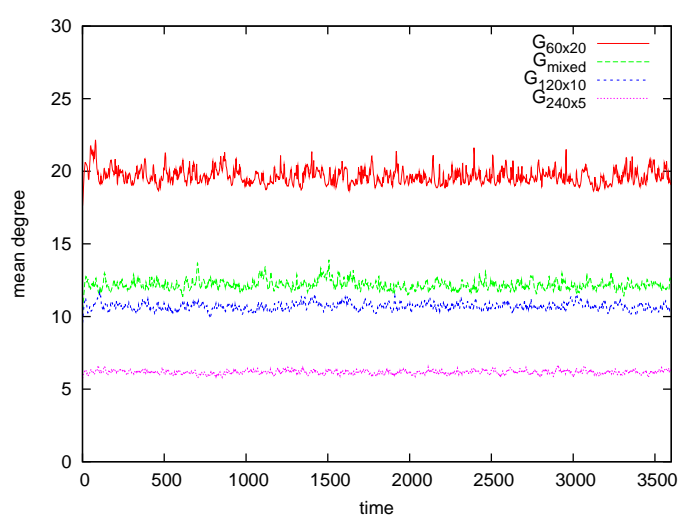

Figure 7. Example of average node degree over time for different group sizes and with a radio range equal to 15 meters.

same degree values. In our model we observe relatively more agents whose degree is close to the mean. This can be explained as the consequence of the use of Levy walk on the mobile agent movements making them almost uniform on a given simulation area (see Figures 4 and 5). For the same reason in our model the agents whose degree is great are less numerous than in the case of the RPGM. A proper modeling of the node degree is an important feature of our model, hence a higher degree allows nodes to have potentially better connectivity.

\subsection{Impact of the Obstacles on Node Degree and Contact/Inter Contact Times for Our Model}

We treat now the graph connectivity in the context of the radio range and groups. The simulation area is a square $1200 \times 1200$ which is free of obstacles. We divide a population of 1200 agents into groups whose radius is 15 meters in four different ways. Three distributions of the population are uniform: 240 teams of 5 nodes, 120 teams of 10 nodes, and 60 teams of 20 nodes. The fourth distribution divides the population into three equal parts of 400 nodes. The first one is divided into 80 teams of 5 nodes, the second one is divided into 40 teams of 10 nodes, and the third one is divided into 20 teams of 20 nodes. In Figure 7 we present variations of the average node degrees in the case when the radio range is equal

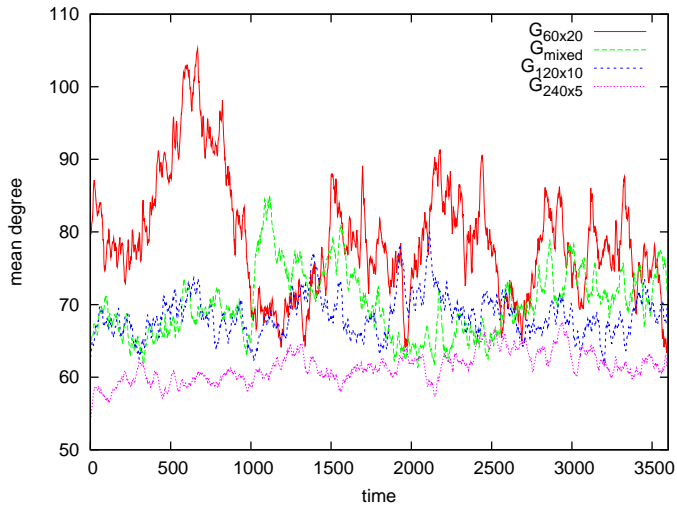

Figure 8. Example of average node degree over time for different group sizes and with a radio range equal to 150 meters.

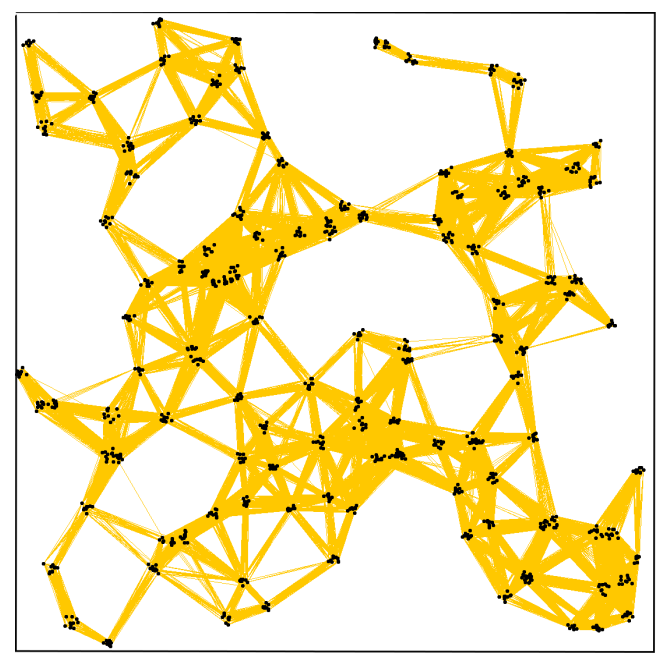

Figure 9. Example of a simulation area for 120 teams of 10 nodes with the 150 meter radio range.

to 15 meters. This hypothesis about the radio range is not realistic. We make, however, this assumption to limit communications to teams' members. Indeed, we observe that the communications between team are rare. A greater variability of the average degree for larger groups emerges from interruptions of connections between members of the same group. When the radio range is realistic, 150 meters, the influence of groups decays (Figure 8). In this case the density of agents becomes the factor determining the graph connectivity (Figure 9).

In the following experiments we use the simulation area map of size $600 \times 600$ with obstacles which is depicted in Figure 10. The influence of obstacles on the average node degree is presented in Figure 11. The obstacles give less freedom to the mobile agents and we observe in this figure that the average node degree is considerably smaller for nodes in the urban environment.

Figure 12 shows the CCDF (Complementary Cumulative Distribution Function, $\mathrm{CCDF}=1-\mathrm{CDF}$ ) representing connection time for nodes controlled by our composite model. The model includes groups of size 10 with a radio range of 175 and the area of simulation both with and without obstacles (urban and rural 




Figure 10. Map used for urban simulations.

scenarii). In the presence of obstacles we observe more short connection times (2-200 seconds) than in the case without obstacles. The obstacles are directly responsible for cutting the connections. As connection time becomes longer, the difference between the environments with and without obstacles disappears because the long connection periods concern principally members of the same team.

The inter-contact time between agents in our model (Figure 13) in the same simulation environment follows a power law as could be expected ([13]). The existence of obstacles makes the inter contact time longer.

\section{Conclusion}

Our goal is to furnish a realistic mobility model in order to validate new routing protocols for ad hoc networks deployed in disaster areas within the context of the RAF project concerning civil protection issues. After surveying the existing mobility models, we decided to propose a new one relating in particular to model displacements of rescue worker teams in the case of action on a disaster scene. We opted for a composite model which takes into account human mobility characteristics, movements of groups of rescue workers,

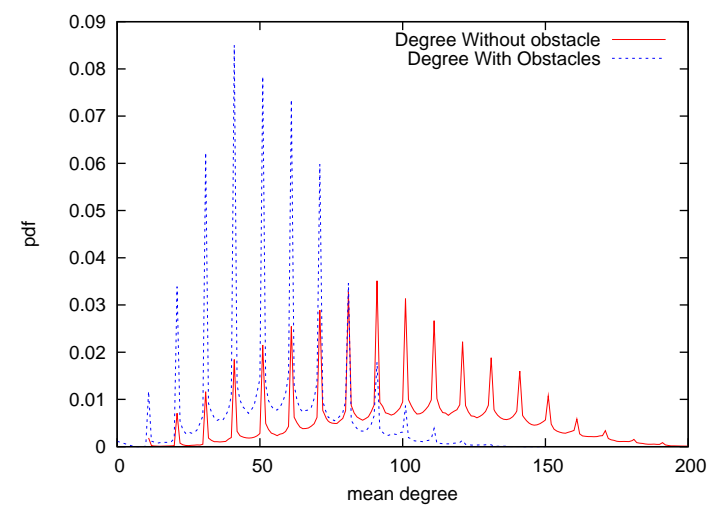

Figure 11. Influence of obstacles on the average node degree.

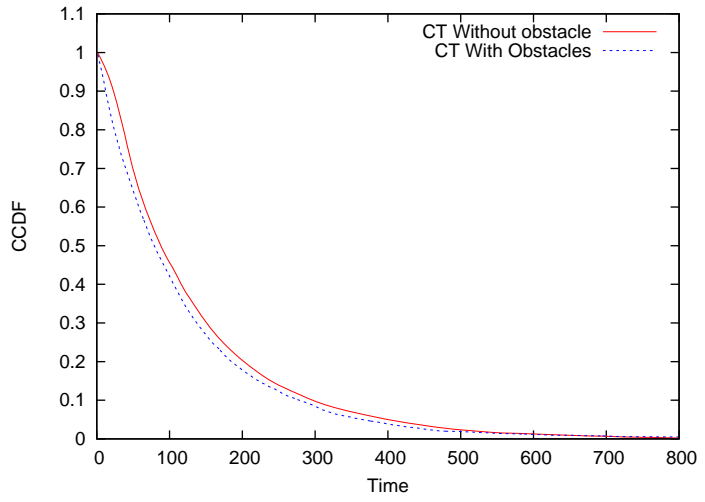

Figure 12. CCDF of Contact Time.



Figure 13. CCDF of Inter Contact Time.

and obstacle avoidance. To this end, we used certain existing models, e.g., the Levy walk model, the RPGM model and the Voronoï diagram, all of which were adapted to operate as parts of our composite model. In order to make the approach resulting from a Voronoï diagram more realistic, we modified the initial choice of reference points.

The evaluation of our model shows that it retains the realism of the Levy-walk and the group mobility of the RPGM while incorporating obstacle avoidance thanks to the Voronoï diagram. Regarding the use of Voronoï diagrams, we propose in this article a new solution, more efficient in circumventing obstacles.

We are also thinking of creating a mobility model on a macroscopic scale, i.e., a model of transport of rescue teams in vehicles to a disaster area. Such a model will need to cope with the different aspects of displacements of actors on the same disaster scene. It could also be used in a certain category of sensor networks called Bioadhoc sensor networks in which humans or animals are equipped with sensors.

\section{ACKNOWLEDGMENTS}

The presented work was done within the context of the RAF project which is also one of the projects of the "SYSTEM@TIC PARIS-REGION competitiveness cluster". SYSTEM@TIC PARIS-REGION and the RAF project are supported by the French Ministry of In- 
dustry, the "departement" Essonne, the "departement" Haut-de-Seines and the Paris General Council.

\section{REFERENCES}

[1] S. Kurkowski, T. Camp, and M. Colagrosso, "MANET simulation studies: The incredibles," ACM SIGMOBILE Mobile Computing and Communications Review, vol. 9, pp. 50-61, 2005.

[2] D. B. Johnson and D. A. Maltz, "Dynamic source routing in ad hoc wireless networks," in Mobile Computing, T. Imielinski and H. F. Korth, Eds. Springer US, 1996, vol. 353 , pp. 153-181.

[3] M. Gyarmati, U. Schilcher, G. Brandner, C. Bettstetter, Y. W. Chung, and Y. H. Kim, "Impact of random mobility on the inhomogeneity of spatial distributions," in Proc. IEEE Global Telecommunications Conference (GLOBECOM), 2008, pp. 404-408.

[4] F. Bai and A. Helmy, Wireless Ad Hoc and Sensor Networks. Kluwer academic Publishers, June 2004, ch. A Survey of Mobility Modeling and Analysis in Wireless Ad Hoc Networks.

[5] C. Bettstetter, "Smooth is better than sharp: A random mobility model for simulation of wireless networks," in Proc. 4th ACM Int. Workshop on Modeling, Analysis and Simulation of Wireless and Mobile Systems (MSWIM). ACM, 2001, pp. 19-27.

[6] E. M. Royer, P. M. Melliar-smith, and L. E. Moser, "An analysis of the optimum node density for ad hoc mobile networks," in Proc. IEEE Int. Conf. Communications (ICC), 2001, pp. 857-861.

[7] N. Aschenbruck, E. Gerhards-Padilla, M. Gerharz, M. Frank, and P. Martini, "Modelling mobility in disaster area scenarios," in Proc. 10th ACM-IEEE Int. Symposium on Modeling, Analysis and Simulation of Wireless and Mobile Systems (MSWIM), 2007.

[8] D. Brockmann, L. Hufnagel, and T. Geisel, "The scaling laws of human travel," Nature, vol. 439, pp. 462-465, 2006.

[9] M. C. Gonzalez, C. A. Hidalgo, and A.-L. Barabasi, "Understanding individual human mobility patterns," Nature, vol. 453, pp. 779-782, June 2008.

[10] I. Rhee, M. Shin, S. Hong, K. Lee, and S. Chong, "On the Levy-Walk nature of human mobility," in Proc. 27th IEEE Conf. Computer Communications (INFOCOM), April 2008, pp. 924-932.

[11] M. Kim, D. Kotz, and S. Kim, "Extracting a mobility model from real user traces," in Proc. 25th Annual Joint Conf. IEEE Computer and Communications Societies (INFOCOM), Barcelona, Spain, April 2006, pp. 1-13.

[12] A. Chaintreau, P. Hui, J. Crowcroft, C. Diot, R. Gass, and J. Scott, "Impact of human mobility on opportunistic forwarding algorithms," IEEE Transactions on Mobile Computing, vol. 6, no. 6, pp. 606-620, 2007.

[13] T. Karagiannis, J.-Y. Le Boudec, and M. Vojnovic, "Power law and exponential decay of inter contact times between mobile devices," in Proc. 13th Annual ACM Int. Conf. Mobile Computing and Networking (MobiCom), 2007.

[14] R. D. Luce and A. D. Perry, "A method of matrix analysis of group structure," Psychometrika, vol. 14, no. 2, pp. 95116, 1949.

[15] X. Hong, M. Gerla, G. Pei, and C. chuan Chiang, "A group mobility model for ad hoc wireless networks," in Proc. 2nd ACM Int. Workshop on Modeling, Analysis and Simulation of Wireless and Mobile Systems (MSWIM), 1999, pp. 53-60.

[16] H. Berlin, Ed., Computational Geometry. Springer, 2008, ch. 15.

[17] A. Jardosh, E. M. Belding-Royer, K. C. Almeroth, and S. Suri, "Towards realistic mobility models for mobile ad hoc networks," in Proc. 9th Annual ACM Int. Conf. Mobile Computing and Networking (MobiCom), 2003, pp. 217-229.
[18] C. Papageorgiou, K. Birkos, T. Dagiuklas, and S. Kotsopoulos, "Simulating mission critical mobile ad hoc networks," in Proc. 4th ACM Workshop Performance Monitoring and Measurement of Heterogeneous Wireless and Wired Networks, New York, NY, USA, 2009, pp. 143-150.

[19] E. W. Dijkstra, "A note on two problems in connexion with graphs," Numerische Mathematik, vol. 1, pp. 269-271, 1959.

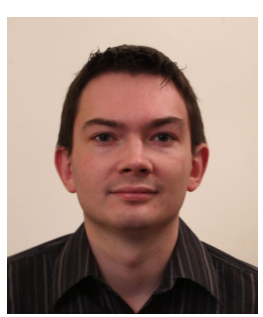

Stéphane Pomportes is a Ph.D. student in Electrical Engineering at the University ParisSud, France and currently works in the field of mobility modeling and resources allocation for ad hoc networks. He received his MS in Combinatorics, Parallel Computing and Knowledge Engineering from the University of Picardie Jules Verne, France in 2008. He worked on the national research project RAF concerning civil security ad hoc networks from 2008 to 2010 . His research interests focus on resource allocation methods for ad hoc networks taking into account the agents' mobility.



Joanna Tomasik received her magisterium diplomas in applied mathematics and in computer science at the University of Technology in Gliwice, Poland, in 1988 and 1989, respectively. She obtained her PhD in 1998 from the same university.

In 1988 she started to work at the Institute of Theoretical and Applied Computer Science of the Polish Academy of Sciences in Gliwice. She spent an academic year as a research fellow at the University of Edinburgh, the UK.

She also worked in France at the University of Versailles and the National Institute of Telecommunications.

Since 2002 Joanna Tomasik has worked permanently in France and she is a professor in the Computer Science Department of SUPELEC, one of the leading French engineering schools. Her main research domains concern performance of computer systems, and in particular of telecommunication networks.

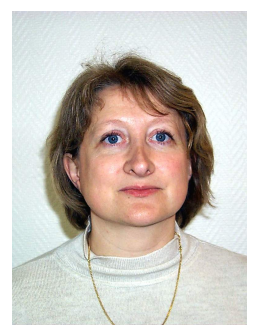

Véronique Vèque $\left(\mathrm{B}^{\prime} 84, \mathrm{MsC}^{\prime} 85\right)$ received a Ph.D. degree in communication networks from University Pierre et Marie Curie (Paris 6) Paris (France), in 1989. From 1989 to 2000, she was an Associate Professor at University of Paris Sud (Paris 11), France and, a research member of Laboratory for Computer Science in Orsay. From 2000 to present, she works as a full Professor at University of Paris Sud and, is a research member of Institute of Electronics. Her research interests lie in the field of both wireless, mobile and high speed communication networks with emphasis on resource allocation, quality of service techniques, ad hoc routing and performance evaluation. She has supervised 11 Ph.D. theses. She was co-author of a book on high-speed networks and ATM techniques in 1995. She has published more than 55 papers in international journals or conferences. Véronique Vèque has been involved in the technical program committee of different IEEE conferences, including GLOBECOM, PIMRC, ISCC, WLN, and WiMOB. She is recently one of the Guest Editors of a special issue on Dynamic Spectrum Management in Wireless Networks of IEEE Wireless Communications journal. Véronique Vèque is also a Senior member of the IEEE, IEEE Communications Society, and IEEE Vehicular Society. 